Supplement of Geosci. Instrum. Method. Data Syst., 6, 311-317, 2017

https://doi.org/10.5194/gi-6-311-2017-supplement

(c) Author(s) 2017. This work is distributed under

the Creative Commons Attribution 3.0 License.

(c) (1)

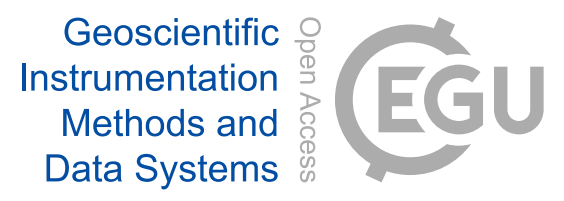

Supplement of

\title{
Time-stamp correction of magnetic observatory data acquired during unavailability of time-synchronization services
}

Pierdavide Coïsson et al.

Correspondence to: Pierdavide Coïsson (coisson@ipgp.fr)

The copyright of individual parts of the supplement might differ from the CC BY 3.0 License. 


\section{Introduction}

The lag between the time series of magnetic components measurements recorded at the magnetic observatories of Lanzhou (LZH) and Kakioka (KAK) have been calculated. As described in the article, 1 hour of data recorded at $1 \mathrm{~Hz}$ sampling frequency have been cross-correlated to compute the lag of the time-stamp of LZH data, after the GPS synchronization of the

5 data logger was unavailable. The local time of the selected data has an impact on the reliability of the computed lag, the hours around local noon being the best choice for the $\mathrm{X}$ component. This is due to the differences in the diurnal variation at LZH and KAK.

Table 1 shows a statistics of lags between LZH and KAK for the period between 1 January 2013 and 7 March 2013, when the GPS time synchronization was still operational. It appears from that the best UTC for checking the time lag between these two 10 observatory is 5:30 UTC, when an average lag of $-3 \mathrm{~s}$ is seen, with a standard deviation of $11 \mathrm{~s}$ on the X component.

Table 2 shows the statistics of the lag between the two acquisition systems of LZH, during the same period. Since the dependence on UTC is not significant, only a global statistics is presented.

Figures 1-24 show the day-to-day evolution of the computed lags between LZH and KAK at every UTC.

Figure 25 shows all the lags between LZH and KAK computed at every UTC. This figure can be compared with Figure 4 of the article. 
Table 1. Statistics of the lag computed between LZH and KAK between 1 January 2013 and 7 March 2013, when LZH GPS synchronization was still operational. The statistic is based on cross-correlations computed with one hour of data centred on the indicated UTC.

\begin{tabular}{lcccccccr}
\hline UTC & mean X & std X & mean Y & std Y & mean Z & std Z & mean F & std F \\
\hline 0.5 & -3.5 & 31.7 & -50.0 & 92.1 & 38.3 & 117.6 & 3.6 & 45.5 \\
1.5 & -6.3 & 25.6 & -31.1 & 116.1 & 51.2 & 89.0 & 5.7 & 22.6 \\
2.5 & -4.5 & 14.3 & -23.2 & 76.9 & 46.5 & 94.2 & 7.2 & 21.4 \\
3.5 & -5.4 & 24.8 & -2.4 & 70.9 & 54.0 & 81.6 & 4.2 & 17.6 \\
4.5 & -4.4 & 13.1 & 42.7 & 77.8 & 40.8 & 91.3 & 4.7 & 16.5 \\
5.5 & -3.1 & 11.6 & 79.0 & 64.5 & 34.9 & 78.9 & 2.5 & 20.8 \\
6.5 & -1.4 & 23.0 & 45.2 & 53.6 & 15.7 & 120.6 & 3.9 & 53.5 \\
7.5 & -7.2 & 50.0 & 8.3 & 62.1 & 34.0 & 108.1 & 1.6 & 54.0 \\
8.5 & -2.3 & 23.5 & -5.2 & 53.2 & 34.1 & 93.3 & 13.7 & 50.3 \\
9.5 & -13.1 & 53.1 & -9.9 & 61.5 & 27.5 & 106.8 & 8.5 & 57.9 \\
10.5 & -17.1 & 61.6 & -25.7 & 77.7 & 12.6 & 86.2 & -3.9 & 51.2 \\
11.5 & -24.5 & 70.2 & -6.5 & 97.0 & 41.3 & 70.0 & -6.7 & 58.1 \\
12.5 & -6.6 & 15.6 & -18.3 & 114.3 & 32.0 & 45.3 & 2.4 & 17.7 \\
13.5 & -7.8 & 14.7 & -43.5 & 104.0 & 31.5 & 70.1 & -0.2 & 20.3 \\
14.5 & -13.8 & 26.4 & -39.3 & 83.3 & 24.4 & 64.7 & -4.3 & 26.9 \\
15.5 & -21.7 & 43.2 & -11.6 & 46.7 & 27.2 & 57.5 & -5.8 & 23.0 \\
16.5 & -19.3 & 28.1 & -26.1 & 56.8 & 26.0 & 45.5 & -6.5 & 23.5 \\
17.5 & -20.2 & 35.1 & -12.9 & 45.7 & 9.1 & 96.9 & -3.3 & 43.0 \\
18.5 & -29.8 & 44.7 & -16.5 & 25.9 & 35.7 & 91.1 & -12.5 & 26.0 \\
19.5 & -22.2 & 46.4 & -3.9 & 98.7 & 10.7 & 113.1 & -16.0 & 57.0 \\
20.5 & -30.8 & 53.7 & -79.0 & 151.8 & 30.0 & 119.5 & -19.0 & 70.7 \\
21.5 & -35.7 & 71.7 & -117.9 & 156.8 & -0.2 & 125.3 & -35.2 & 86.1 \\
22.5 & -3.3 & 101.8 & -88.3 & 100.9 & 69.2 & 129.8 & 14.0 & 101.5 \\
23.5 & -11.7 & 96.3 & -69.6 & 86.9 & 11.7 & 131.5 & -1.3 & 81.8 \\
\hline
\end{tabular}

Table 2. Statistics of the lag computed between the two acquisition systems of LZH between 1 January 2013 and 7 March 2013 , when LZH GPS synchronization was still operational. The statistic is based on cross-correlations computed with one hour of data, for every our of each day.

\begin{tabular}{ccccccccr}
\hline UTC & mean X & std X & mean Y & std Y & mean Z & std Z & mean F & std F \\
\hline $0-24$ & 0.1 & 0.3 & 0.1 & 0.3 & 0.1 & 0.3 & -1.4 & 0.8 \\
\hline
\end{tabular}



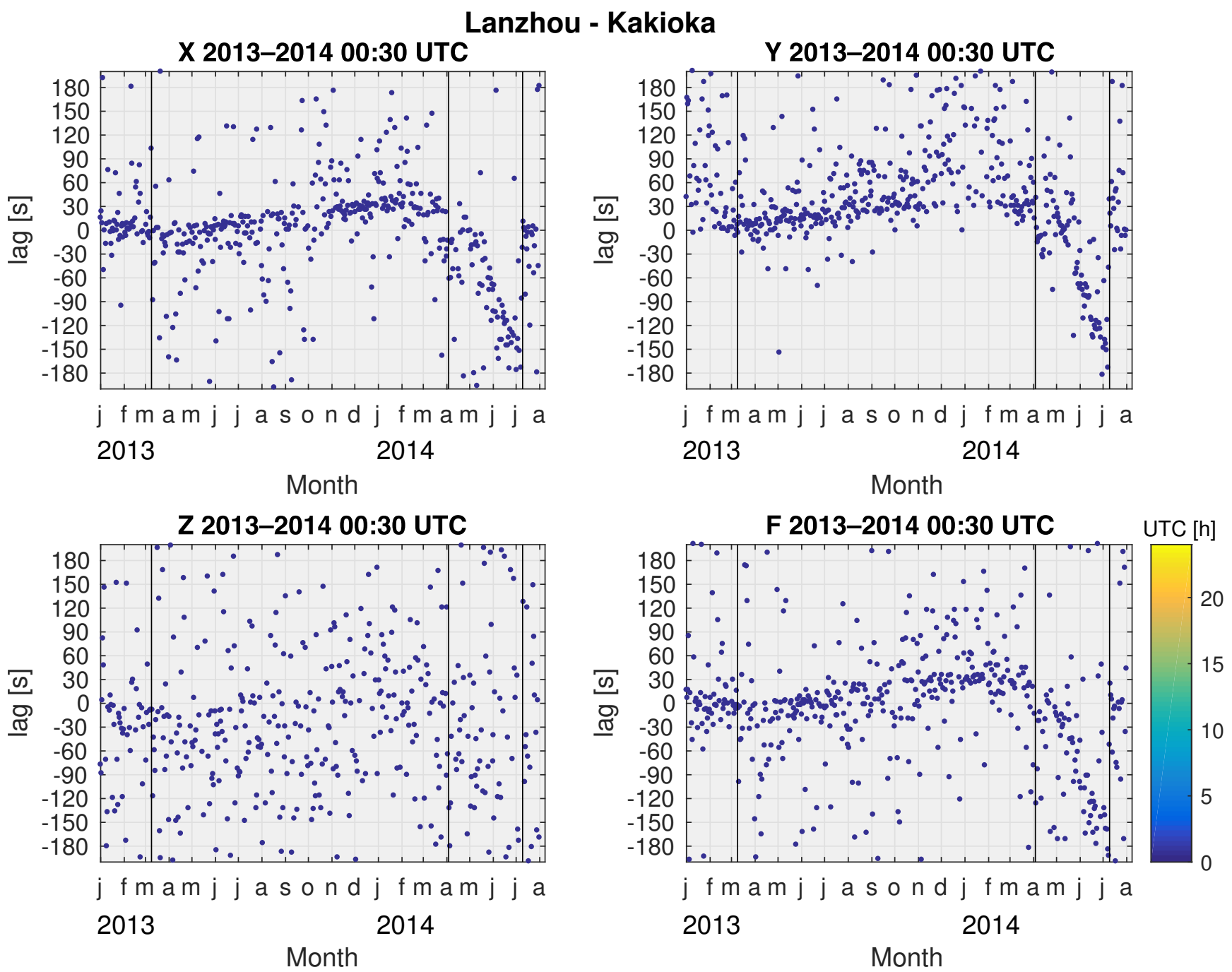

Figure 1. Time lags calculated every day during 1 hour around 00:30 UTC comparing Lanzhou and Kakioka data for each component of the magnetic field X, Y, Z and F. The vertical lines indicate from left to right: the time when the GPS synchronization became unavailable, the time when the data logger was rebooted and the time when the GPS synchronization was re-established. 

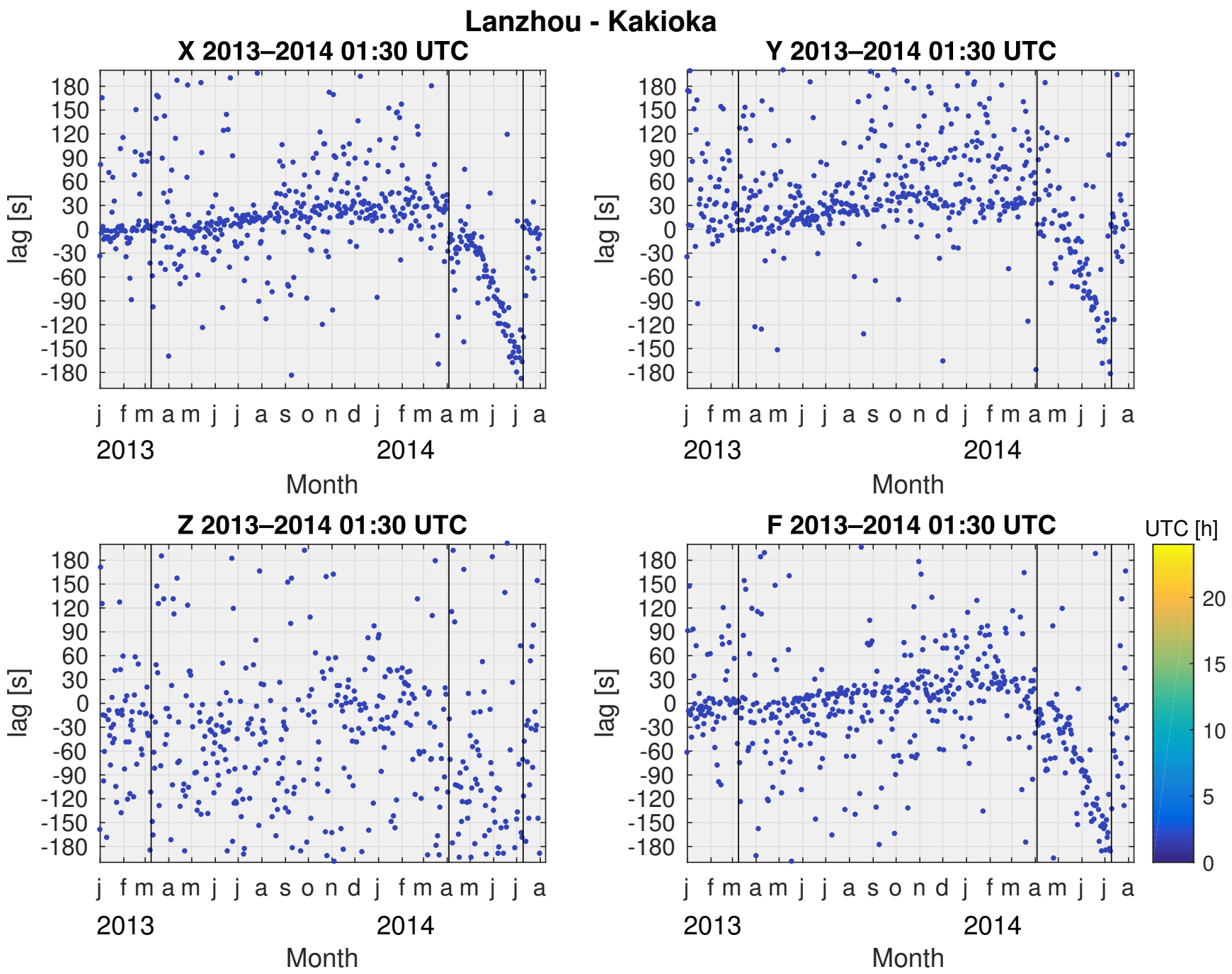

Figure 2. Time lags calculated every day during 1 hour around 01:30 UTC comparing Lanzhou and Kakioka data for each component of the magnetic field X, Y, Z and F. The vertical lines indicate from left to right: the time when the GPS synchronization became unavailable, the time when the data logger was rebooted and the time when the GPS synchronization was re-established. 

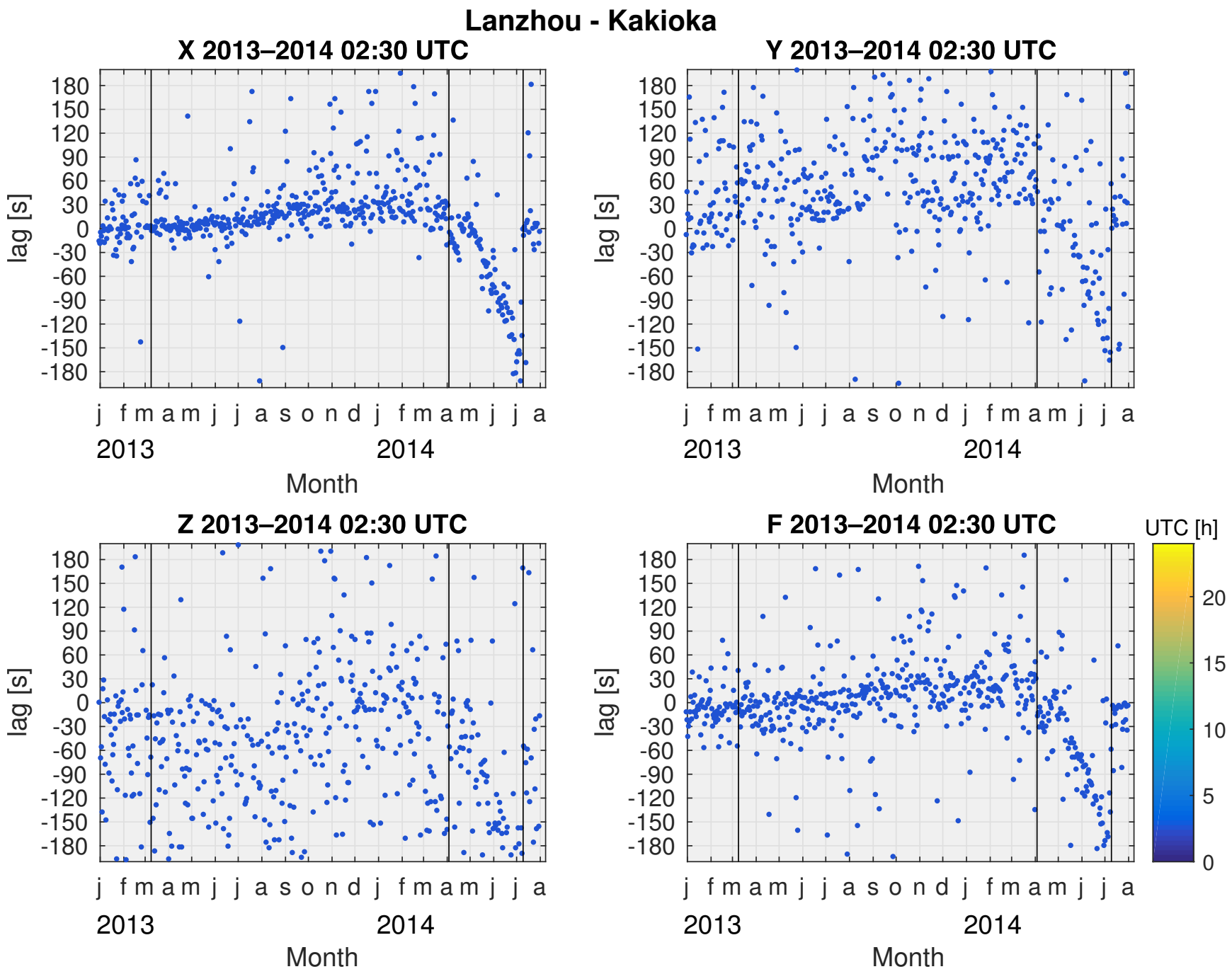

Figure 3. Time lags calculated every day during 1 hour around 02:30 UTC comparing Lanzhou and Kakioka data for each component of the magnetic field X, Y, Z and F. The vertical lines indicate from left to right: the time when the GPS synchronization became unavailable, the time when the data logger was rebooted and the time when the GPS synchronization was re-established. 

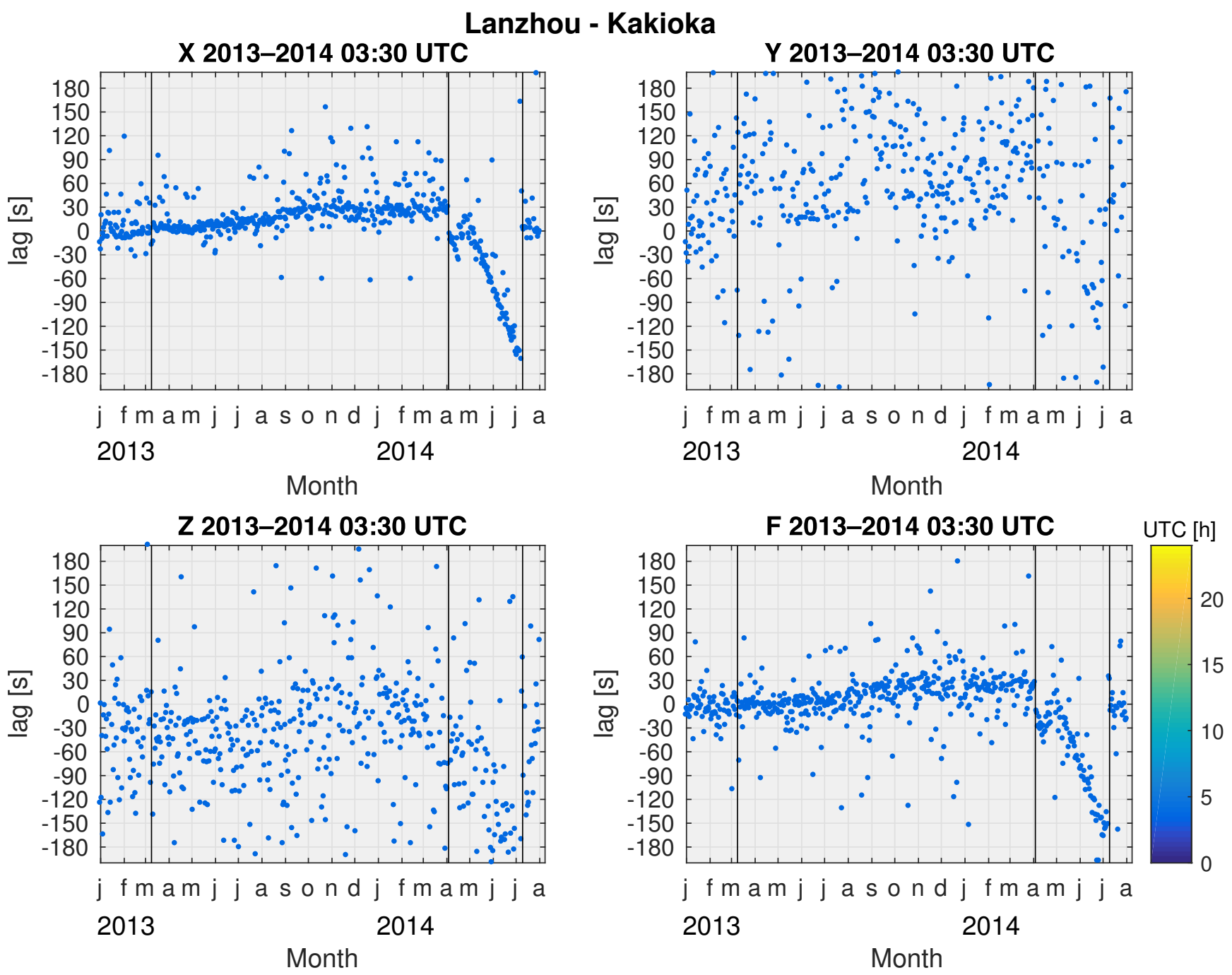

Figure 4. Time lags calculated every day during 1 hour around 03:30 UTC comparing Lanzhou and Kakioka data for each component of the magnetic field X, Y, Z and F. The vertical lines indicate from left to right: the time when the GPS synchronization became unavailable, the time when the data logger was rebooted and the time when the GPS synchronization was re-established. 

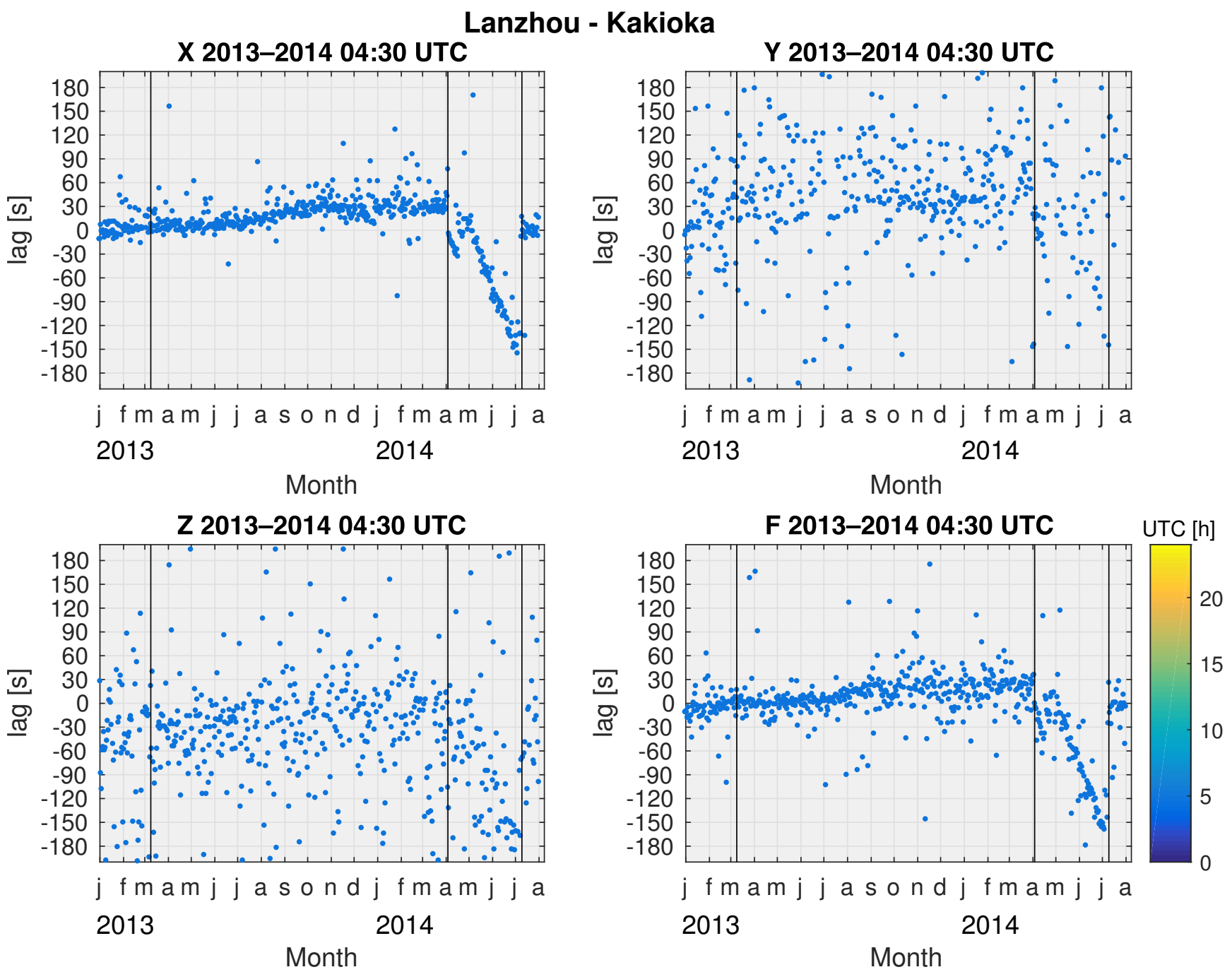

Figure 5. Time lags calculated every day during 1 hour around 04:30 UTC comparing Lanzhou and Kakioka data for each component of the magnetic field X, Y, Z and F. The vertical lines indicate from left to right: the time when the GPS synchronization became unavailable, the time when the data logger was rebooted and the time when the GPS synchronization was re-established. 



Figure 6. Time lags calculated every day during 1 hour around 05:30 UTC comparing Lanzhou and Kakioka data for each component of the magnetic field X, Y, Z and F. The vertical lines indicate from left to right: the time when the GPS synchronization became unavailable, the time when the data logger was rebooted and the time when the GPS synchronization was re-established. 

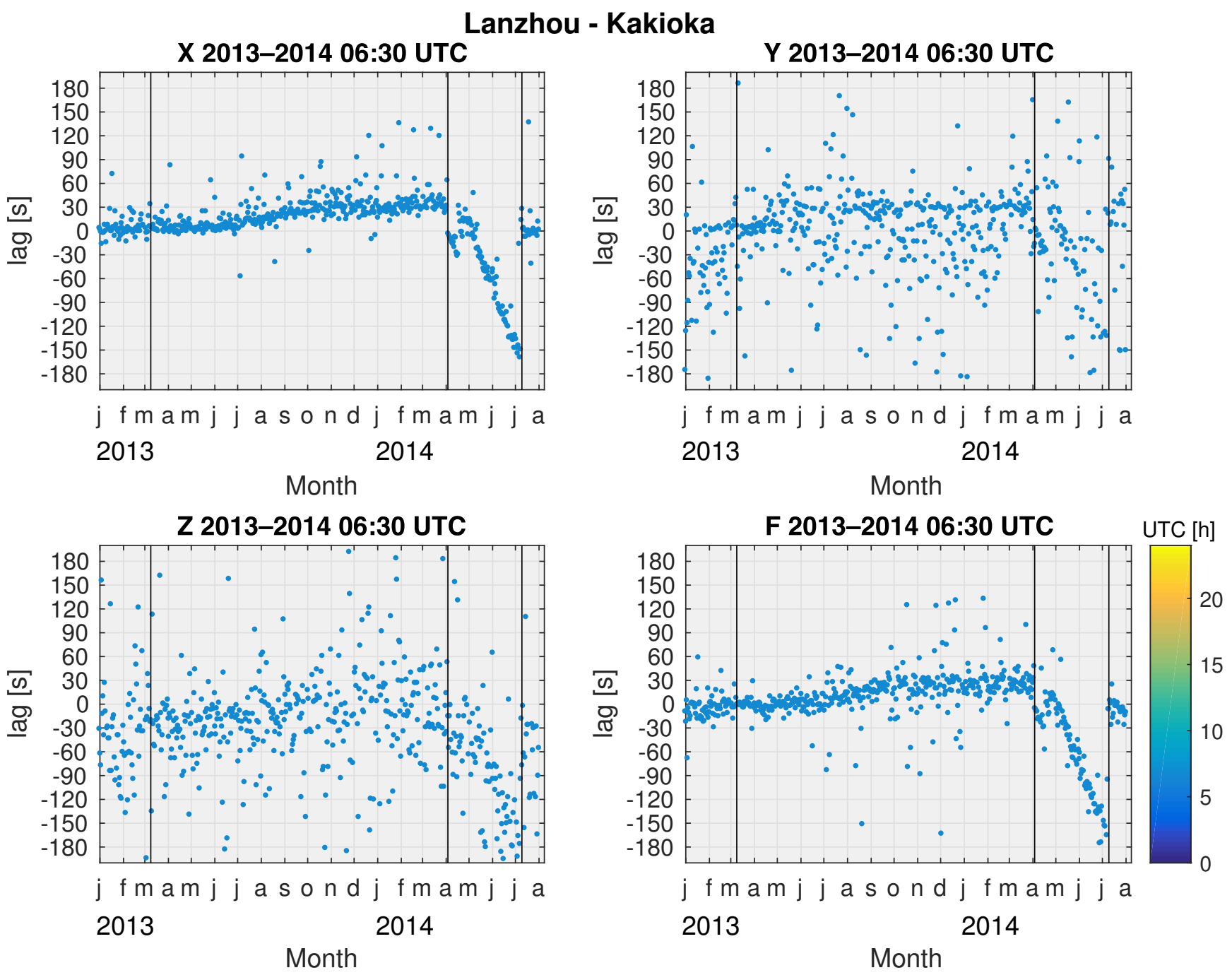

Figure 7. Time lags calculated every day during 1 hour around 06:30 UTC comparing Lanzhou and Kakioka data for each component of the magnetic field X, Y, Z and F. The vertical lines indicate from left to right: the time when the GPS synchronization became unavailable, the time when the data logger was rebooted and the time when the GPS synchronization was re-established. 

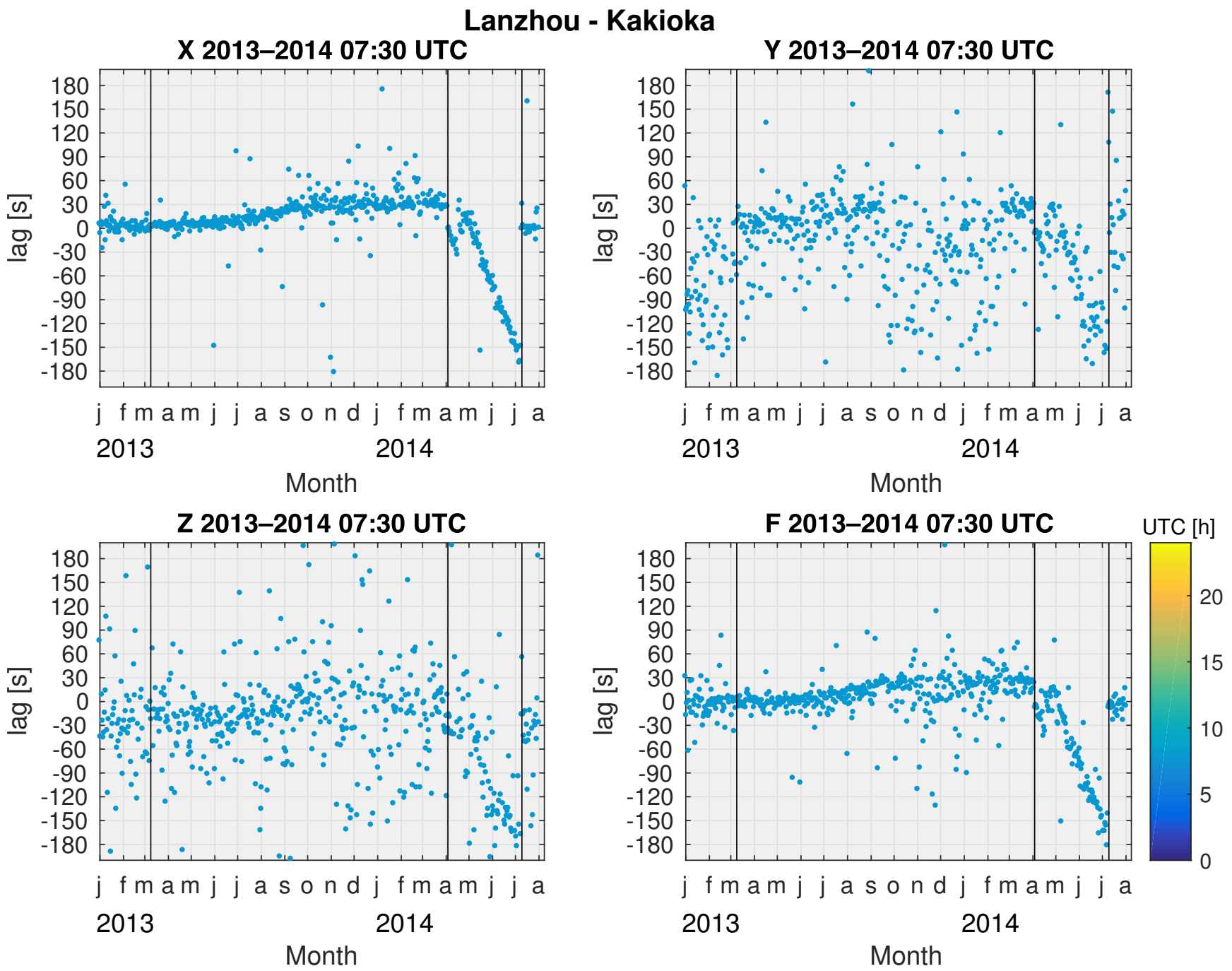

Figure 8. Time lags calculated every day during 1 hour around 07:30 UTC comparing Lanzhou and Kakioka data for each component of the magnetic field X, Y, Z and F. The vertical lines indicate from left to right: the time when the GPS synchronization became unavailable, the time when the data logger was rebooted and the time when the GPS synchronization was re-established. 

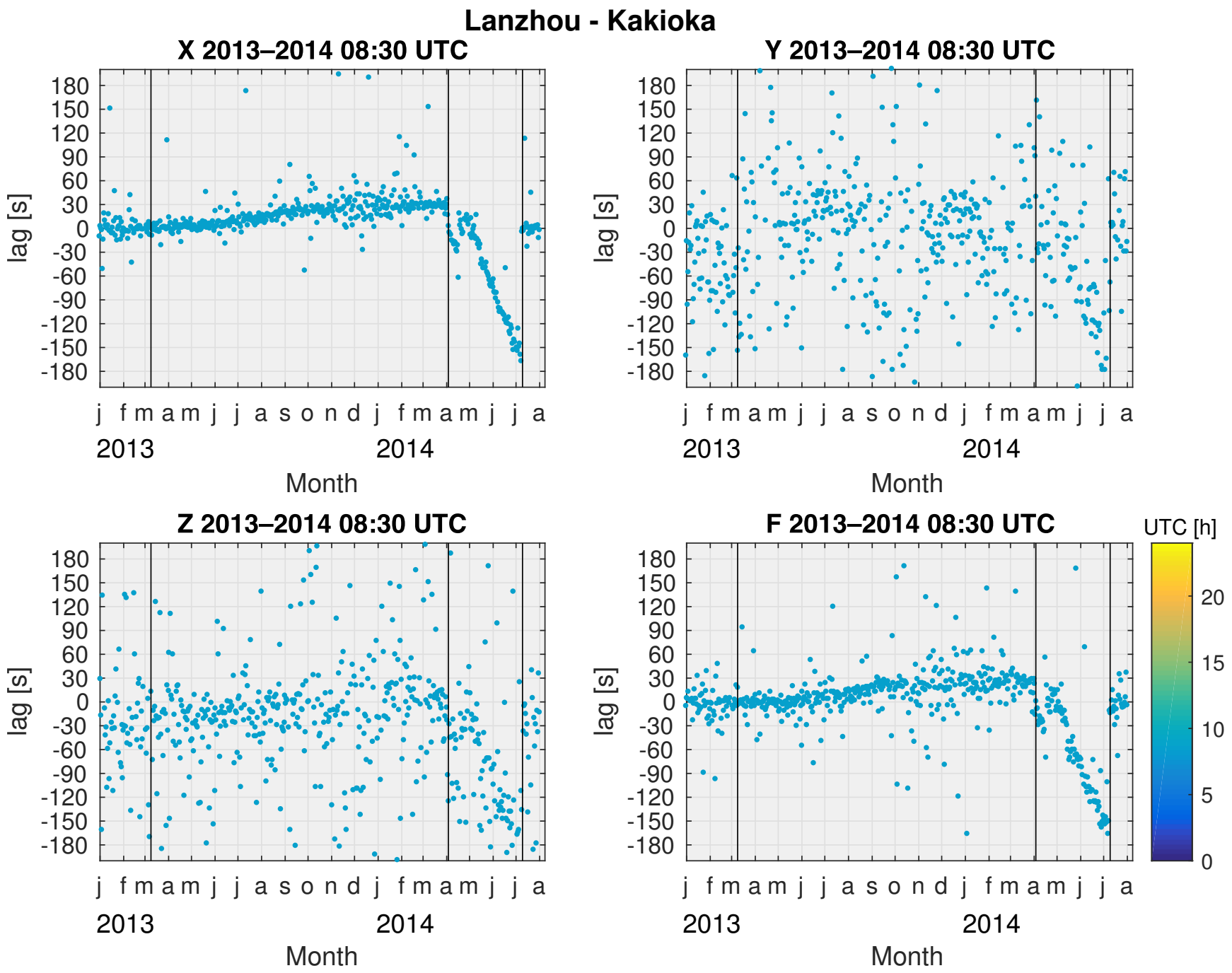

Figure 9. Time lags calculated every day during 1 hour around 08:30 UTC comparing Lanzhou and Kakioka data for each component of the magnetic field X, Y, Z and F. The vertical lines indicate from left to right: the time when the GPS synchronization became unavailable, the time when the data logger was rebooted and the time when the GPS synchronization was re-established. 

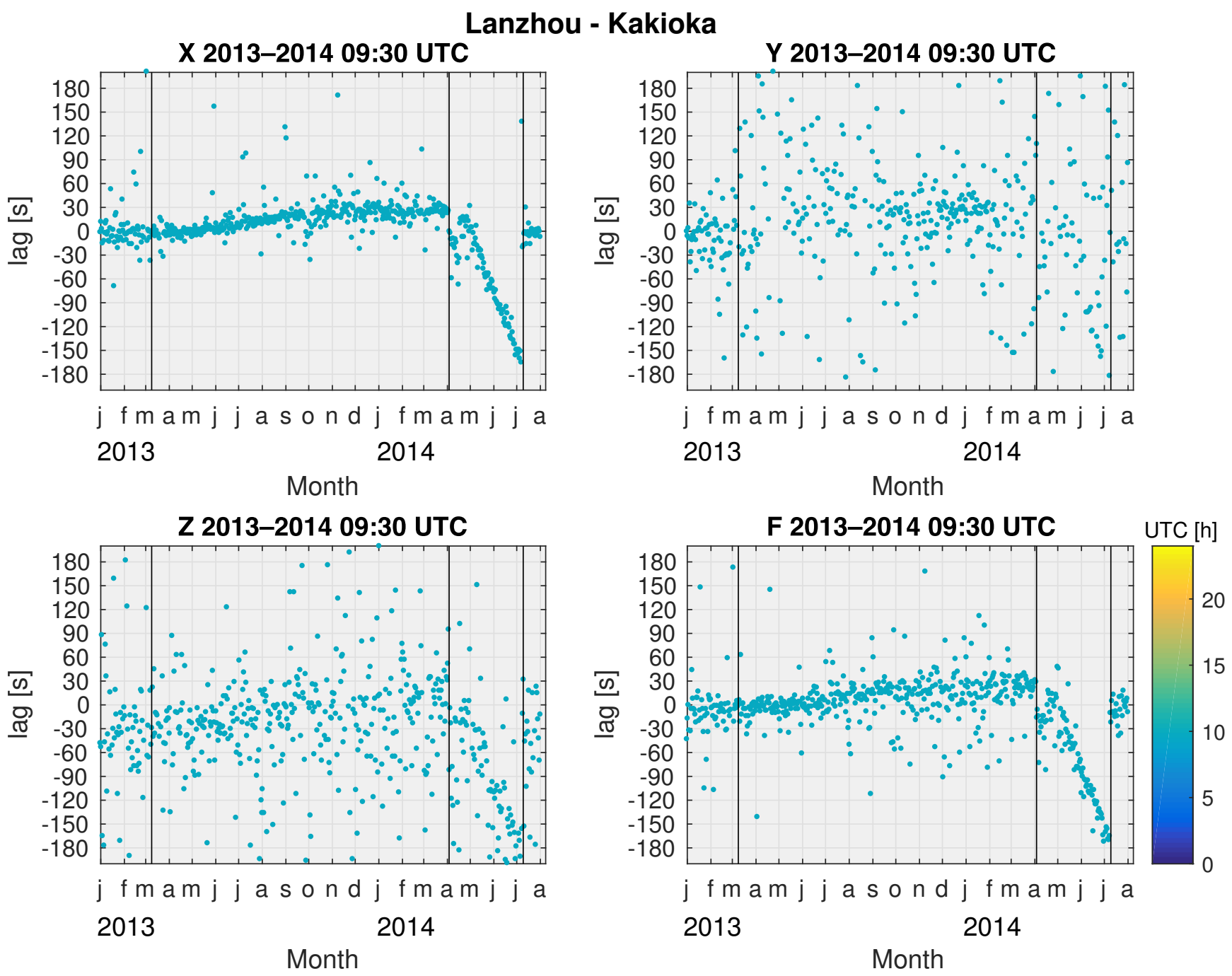

Figure 10. Time lags calculated every day during 1 hour around 09:30 UTC comparing Lanzhou and Kakioka data for each component of the magnetic field X, Y, Z and F. The vertical lines indicate from left to right: the time when the GPS synchronization became unavailable, the time when the data logger was rebooted and the time when the GPS synchronization was re-established. 

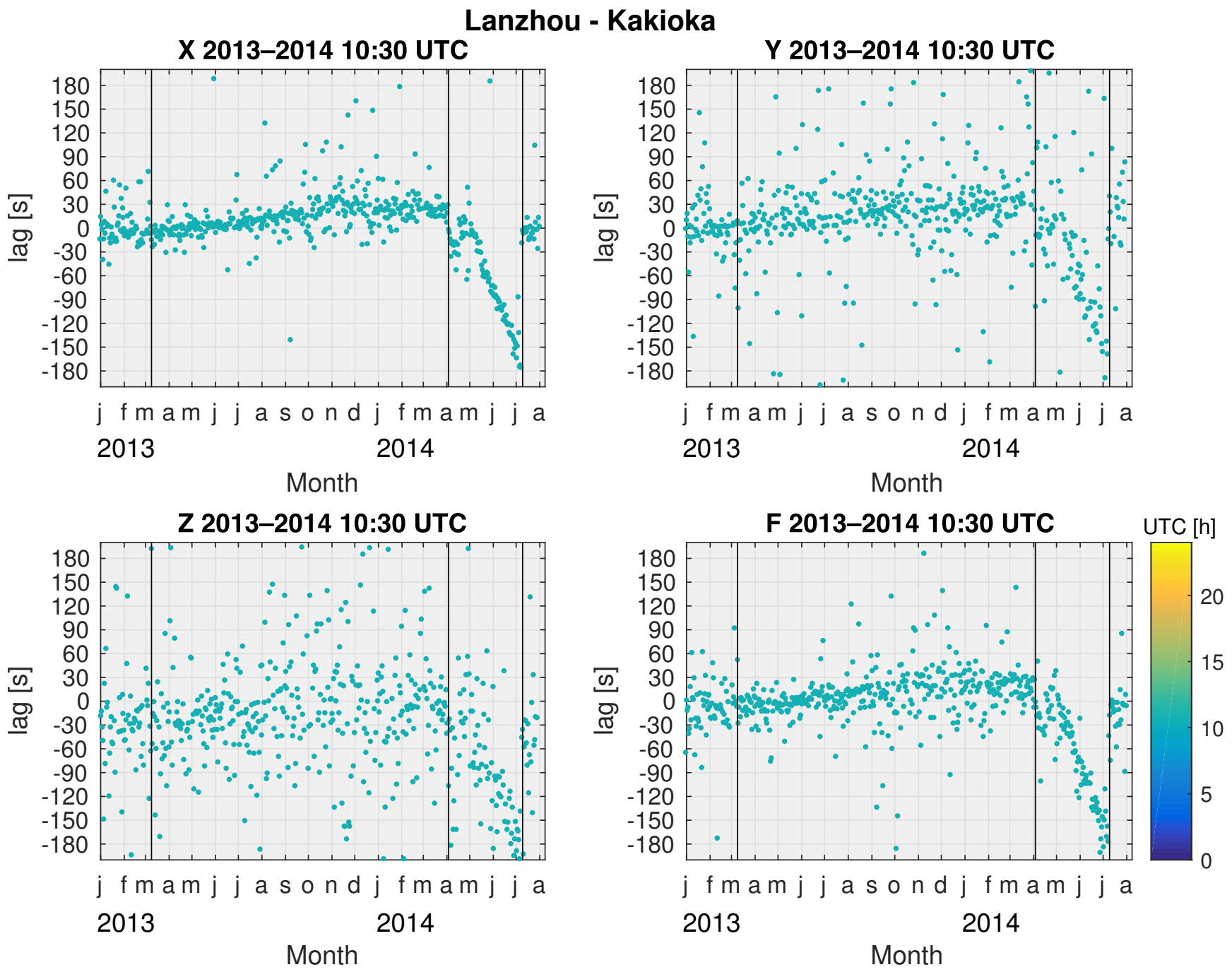

Figure 11. Time lags calculated every day during 1 hour around 10:30 UTC comparing Lanzhou and Kakioka data for each component of the magnetic field X, Y, Z and F. The vertical lines indicate from left to right: the time when the GPS synchronization became unavailable, the time when the data logger was rebooted and the time when the GPS synchronization was re-established. 

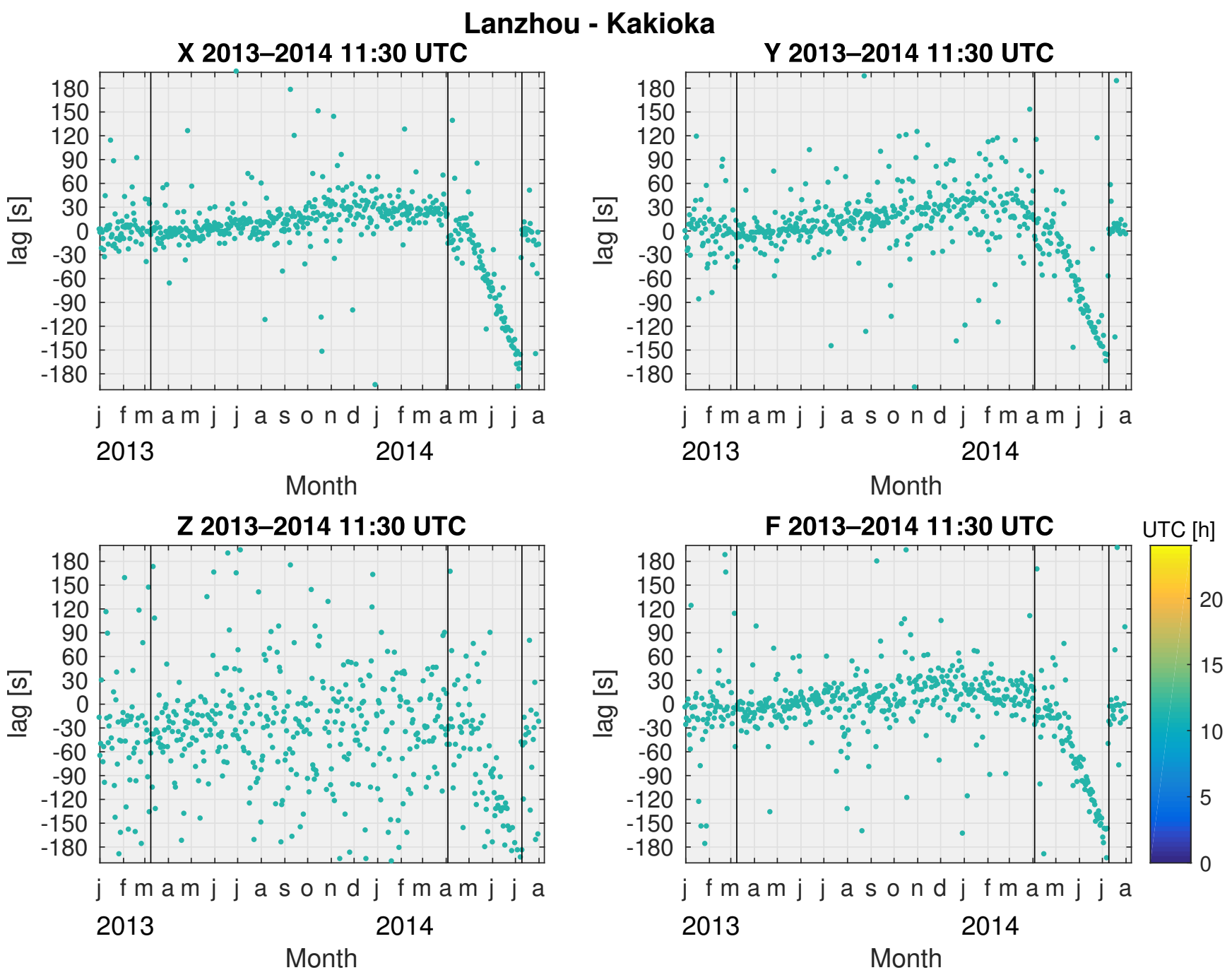

Figure 12. Time lags calculated every day during 1 hour around 11:30 UTC comparing Lanzhou and Kakioka data for each component of the magnetic field X, Y, Z and F. The vertical lines indicate from left to right: the time when the GPS synchronization became unavailable, the time when the data logger was rebooted and the time when the GPS synchronization was re-established. 

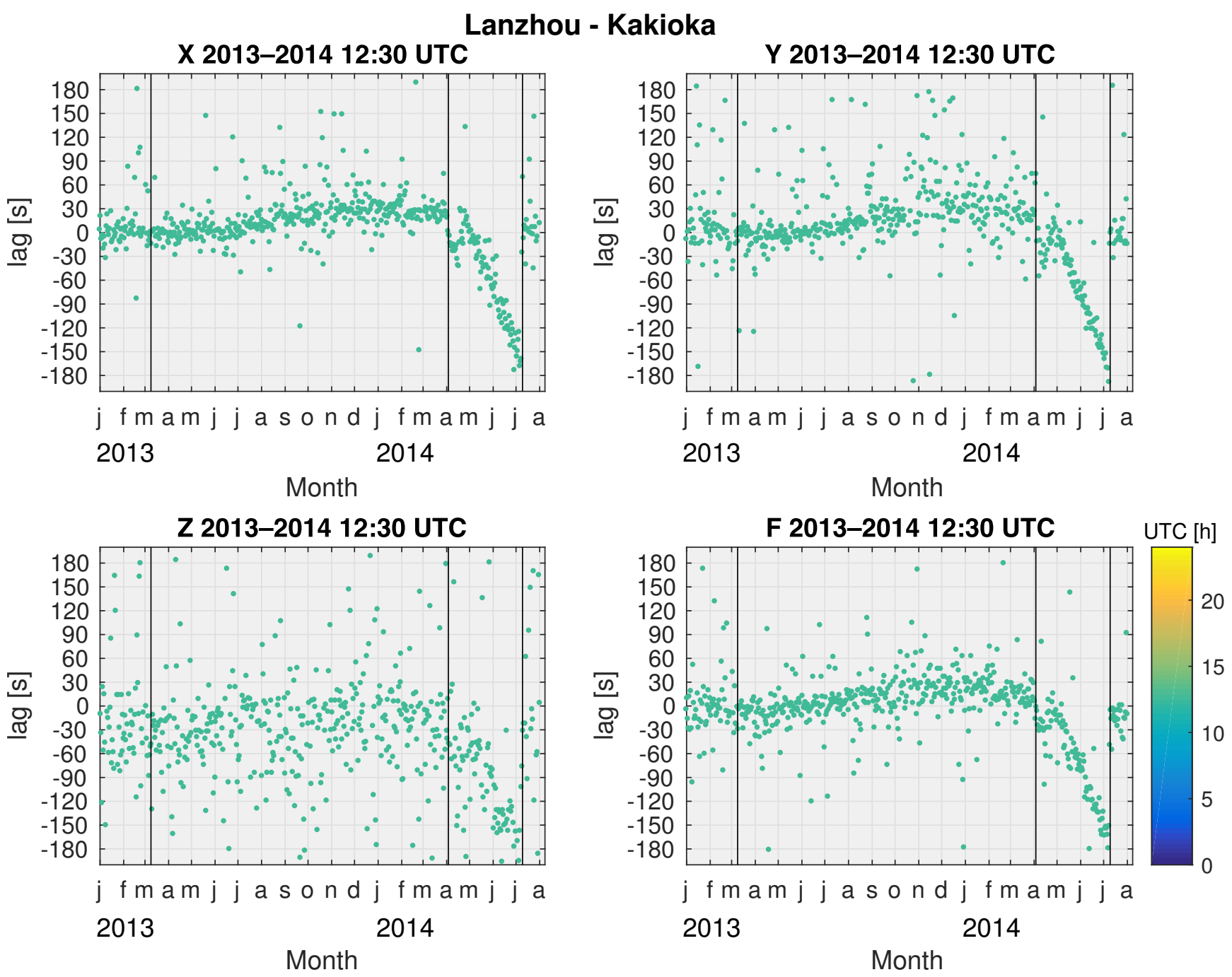

Figure 13. Time lags calculated every day during 1 hour around 12:30 UTC comparing Lanzhou and Kakioka data for each component of the magnetic field X, Y, Z and F. The vertical lines indicate from left to right: the time when the GPS synchronization became unavailable, the time when the data logger was rebooted and the time when the GPS synchronization was re-established. 

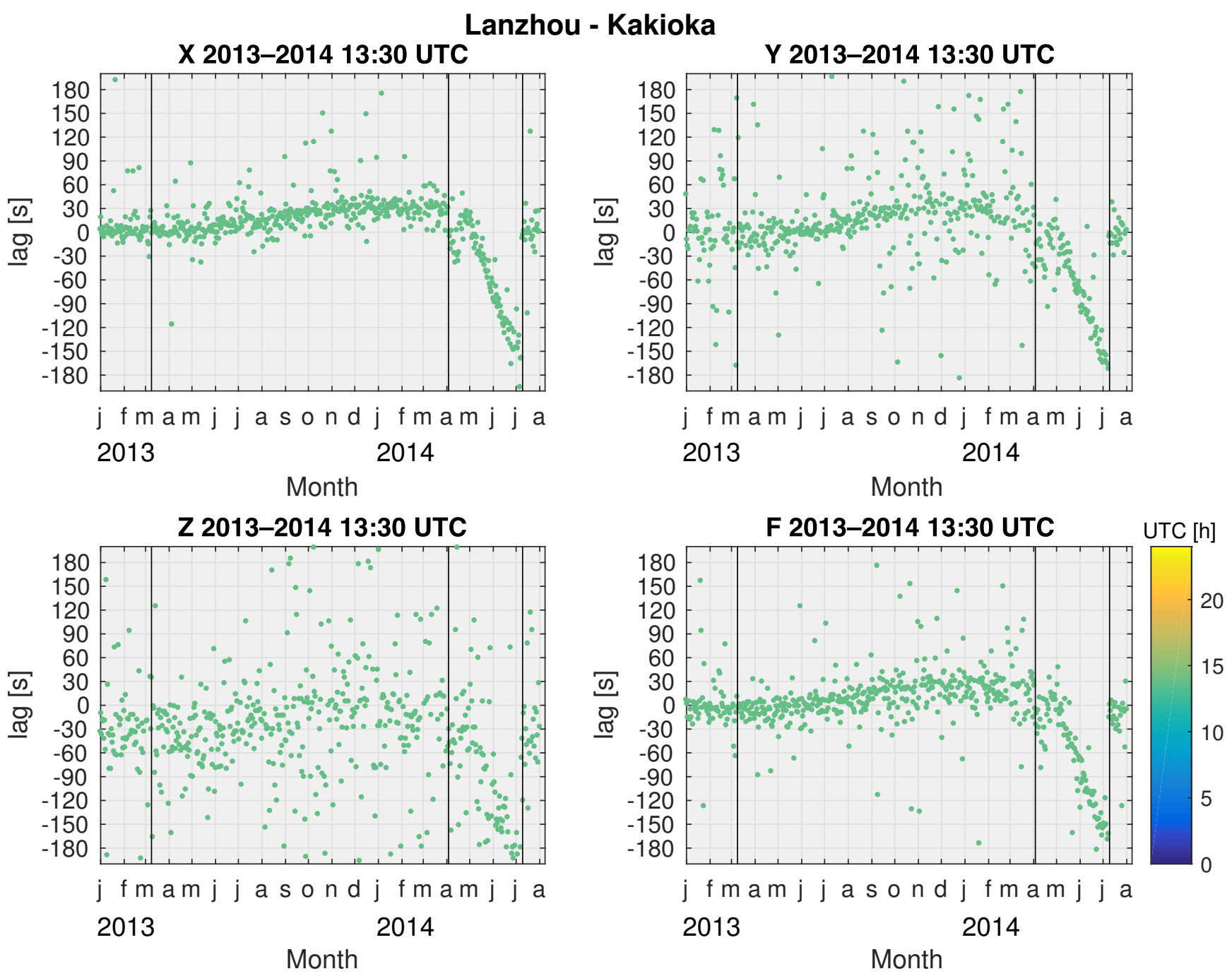

Figure 14. Time lags calculated every day during 1 hour around 13:30 UTC comparing Lanzhou and Kakioka data for each component of the magnetic field X, Y, Z and F. The vertical lines indicate from left to right: the time when the GPS synchronization became unavailable, the time when the data logger was rebooted and the time when the GPS synchronization was re-established. 

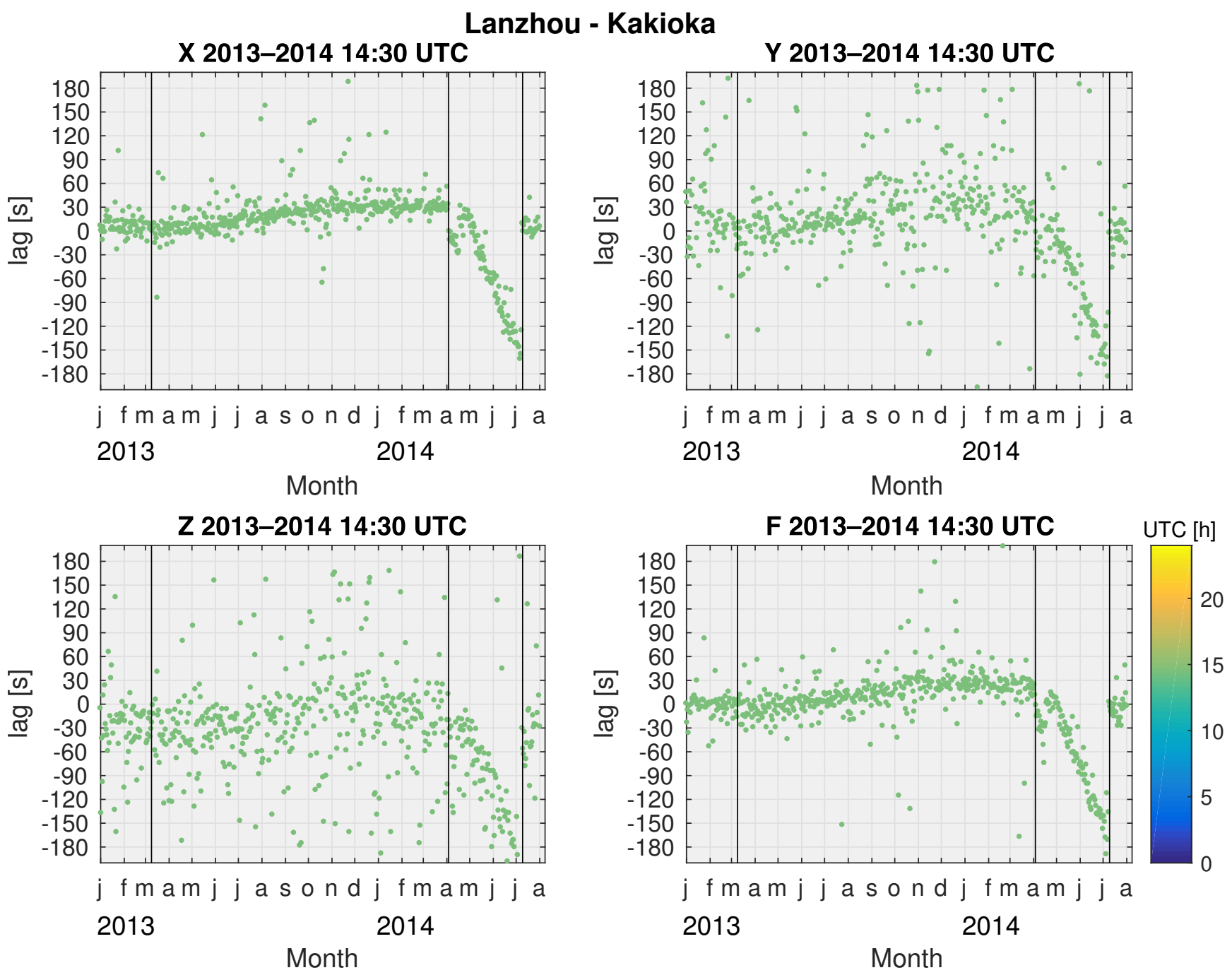

Figure 15. Time lags calculated every day during 1 hour around 14:30 UTC comparing Lanzhou and Kakioka data for each component of the magnetic field X, Y, Z and F. The vertical lines indicate from left to right: the time when the GPS synchronization became unavailable, the time when the data logger was rebooted and the time when the GPS synchronization was re-established. 

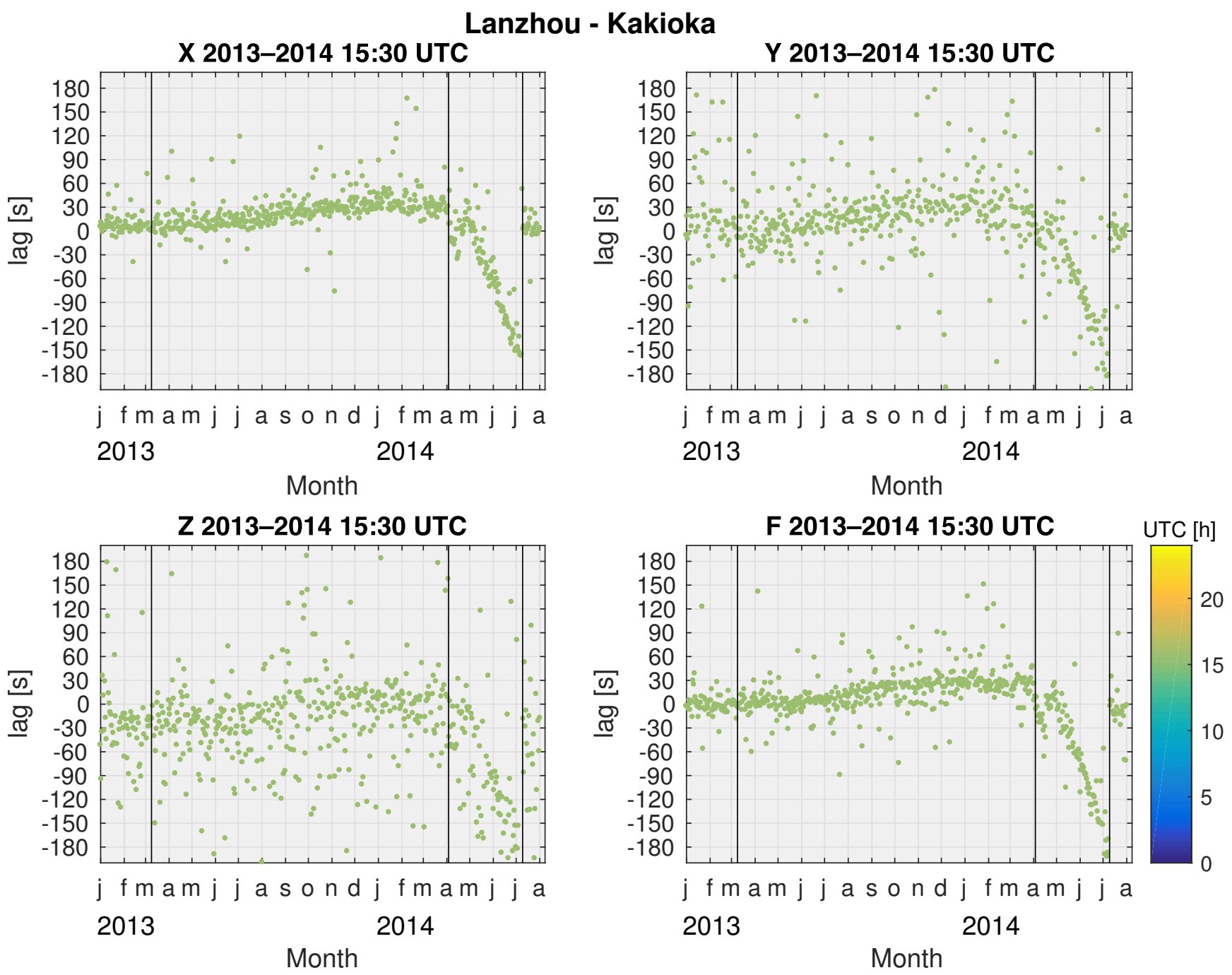

Figure 16. Time lags calculated every day during 1 hour around 15:30 UTC comparing Lanzhou and Kakioka data for each component of the magnetic field X, Y, Z and F. The vertical lines indicate from left to right: the time when the GPS synchronization became unavailable, the time when the data logger was rebooted and the time when the GPS synchronization was re-established. 

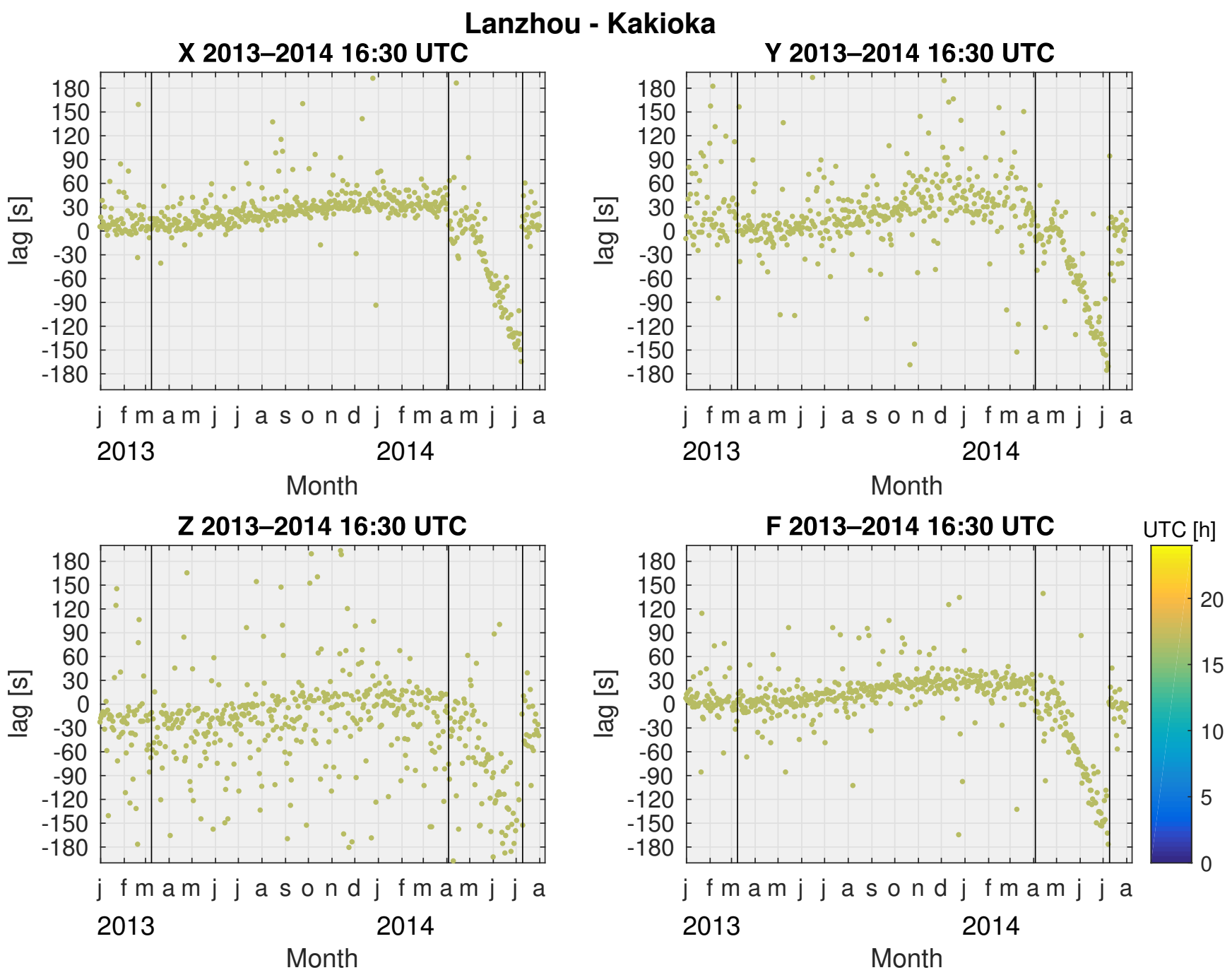

Figure 17. Time lags calculated every day during 1 hour around 16:30 UTC comparing Lanzhou and Kakioka data for each component of the magnetic field X, Y, Z and F. The vertical lines indicate from left to right: the time when the GPS synchronization became unavailable, the time when the data logger was rebooted and the time when the GPS synchronization was re-established. 

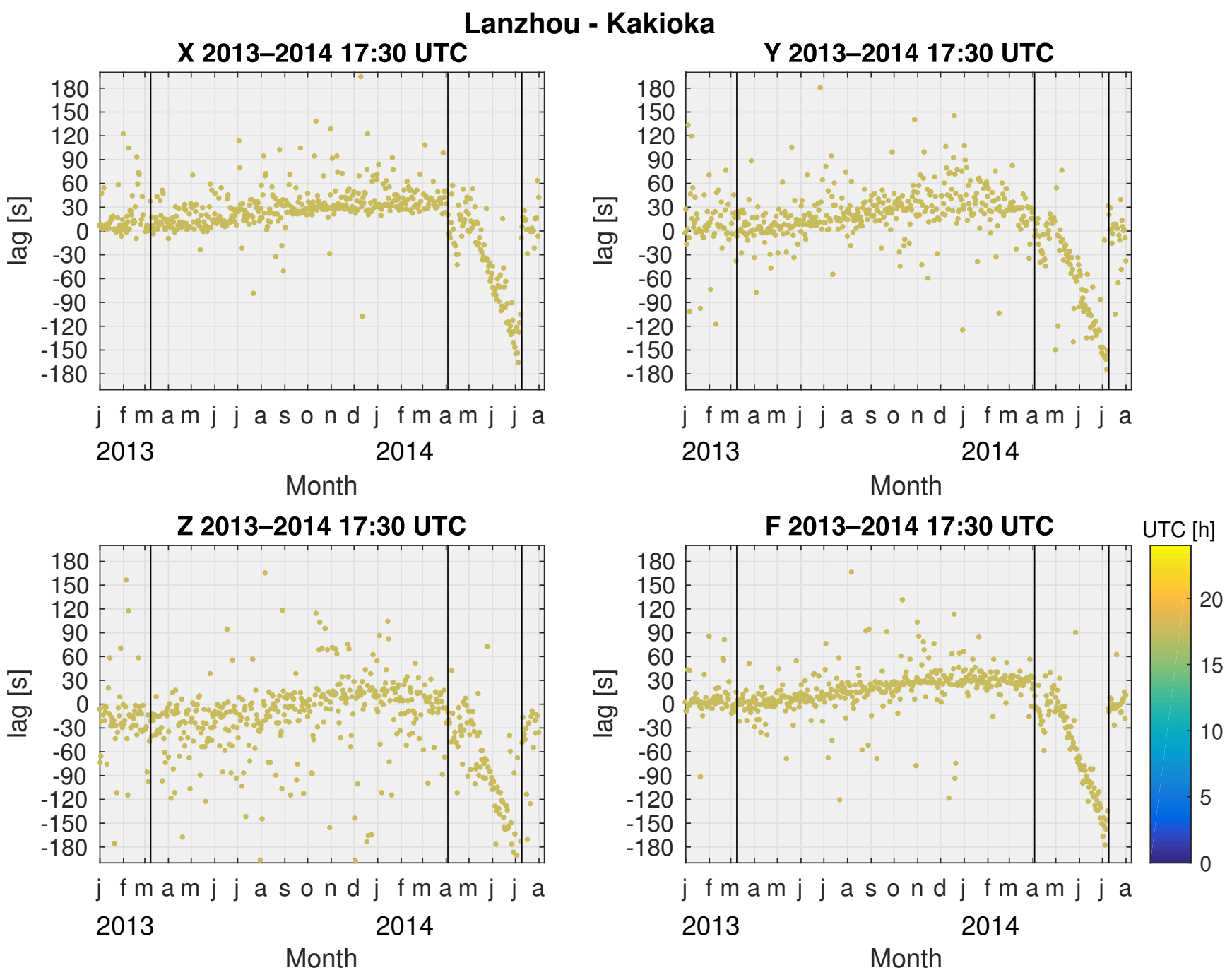

Figure 18. Time lags calculated every day during 1 hour around 17:30 UTC comparing Lanzhou and Kakioka data for each component of the magnetic field X, Y, Z and F. The vertical lines indicate from left to right: the time when the GPS synchronization became unavailable, the time when the data logger was rebooted and the time when the GPS synchronization was re-established. 

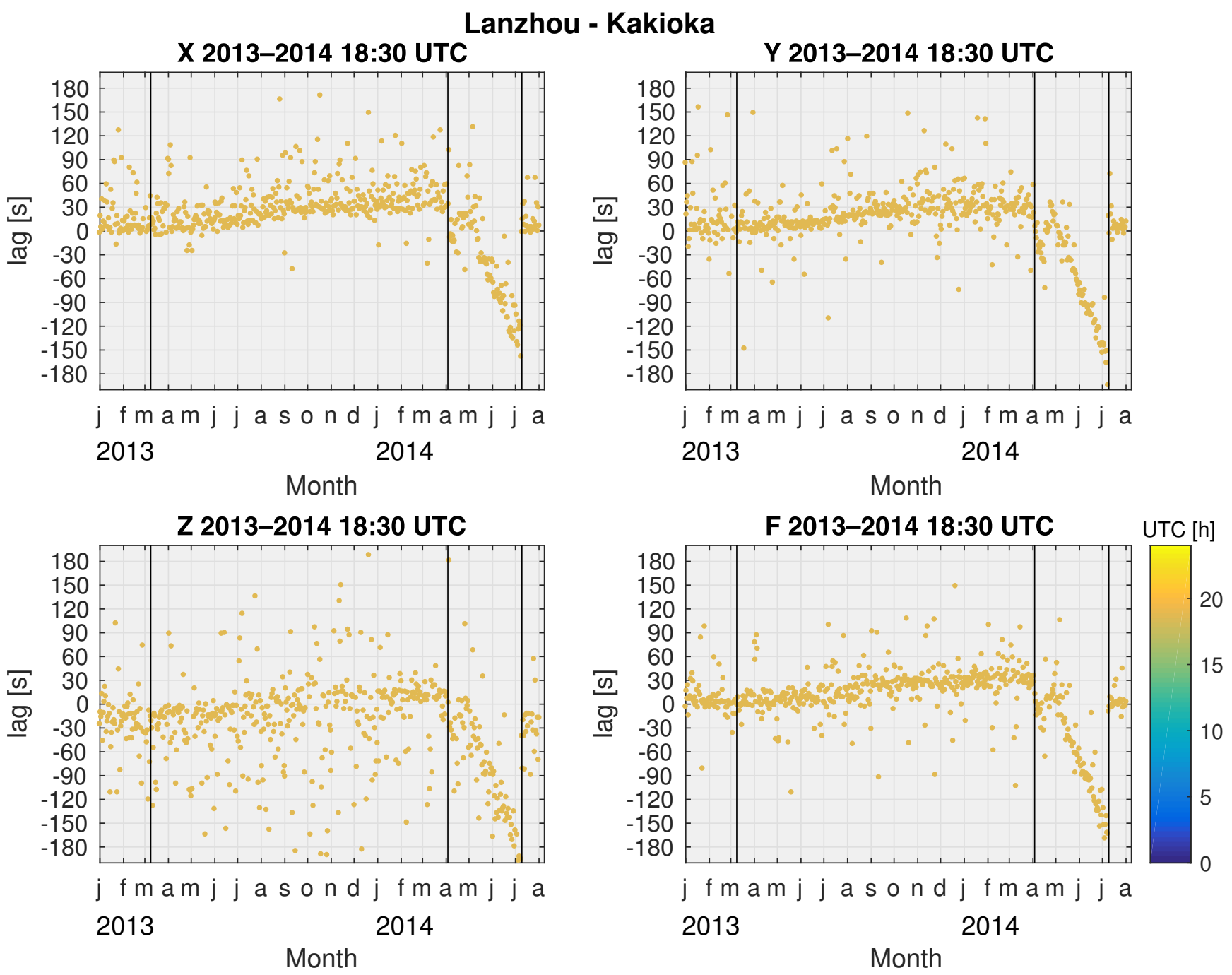

Figure 19. Time lags calculated every day during 1 hour around 18:30 UTC comparing Lanzhou and Kakioka data for each component of the magnetic field X, Y, Z and F. The vertical lines indicate from left to right: the time when the GPS synchronization became unavailable, the time when the data logger was rebooted and the time when the GPS synchronization was re-established. 

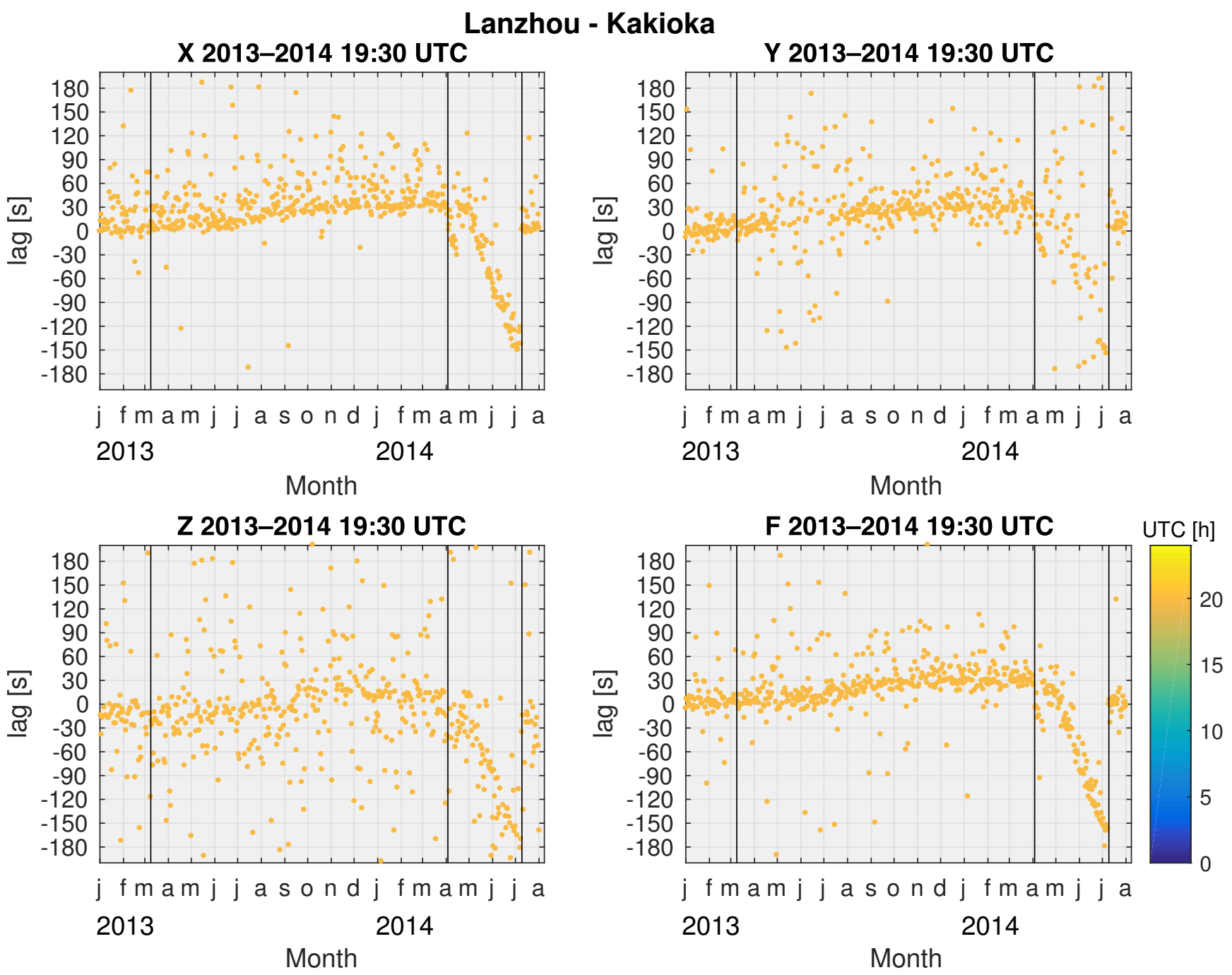

Figure 20. Time lags calculated every day during 1 hour around 19:30 UTC comparing Lanzhou and Kakioka data for each component of the magnetic field X, Y, Z and F. The vertical lines indicate from left to right: the time when the GPS synchronization became unavailable, the time when the data logger was rebooted and the time when the GPS synchronization was re-established. 

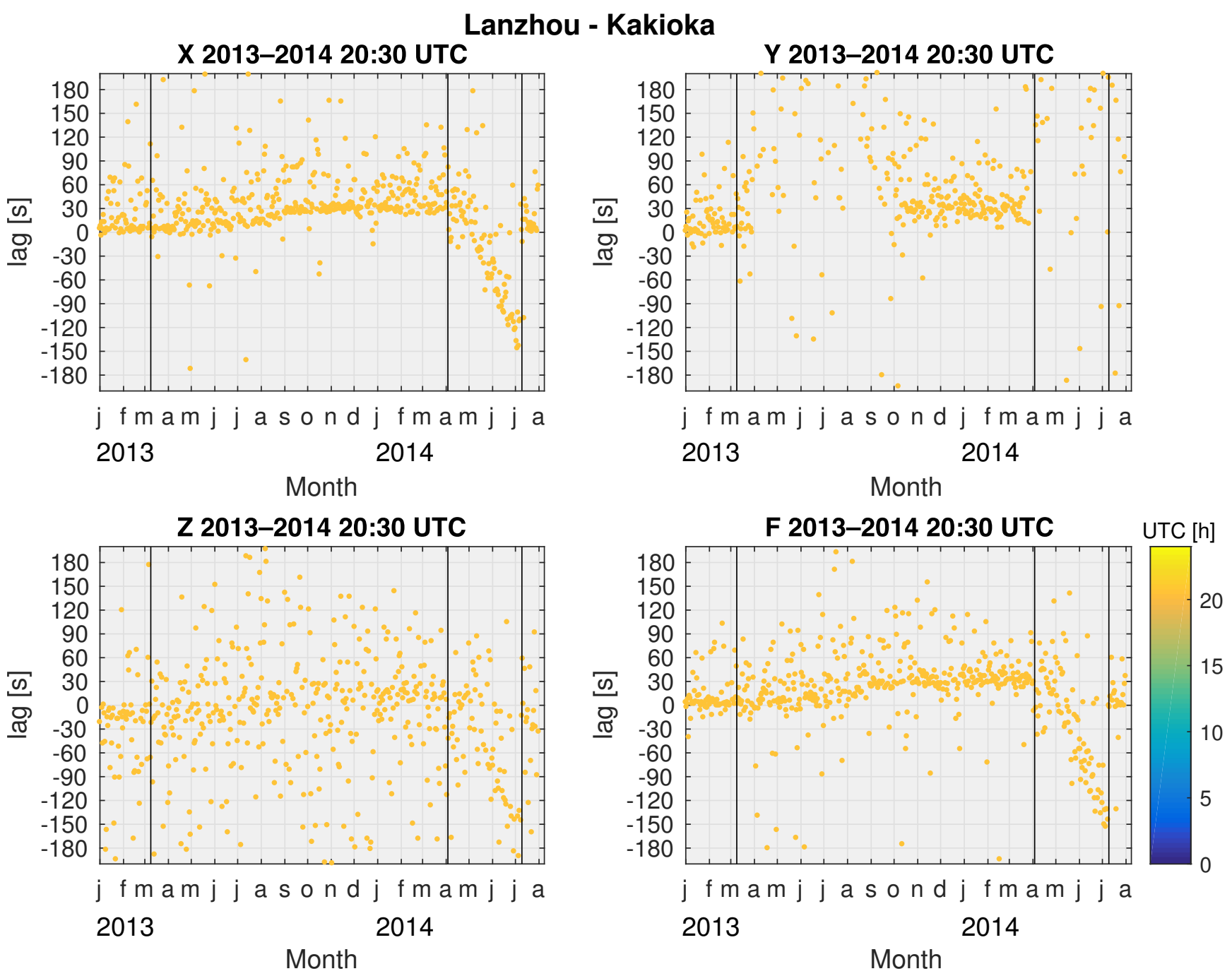

Figure 21. Time lags calculated every day during 1 hour around 20:30 UTC comparing Lanzhou and Kakioka data for each component of the magnetic field X, Y, Z and F. The vertical lines indicate from left to right: the time when the GPS synchronization became unavailable, the time when the data logger was rebooted and the time when the GPS synchronization was re-established. 

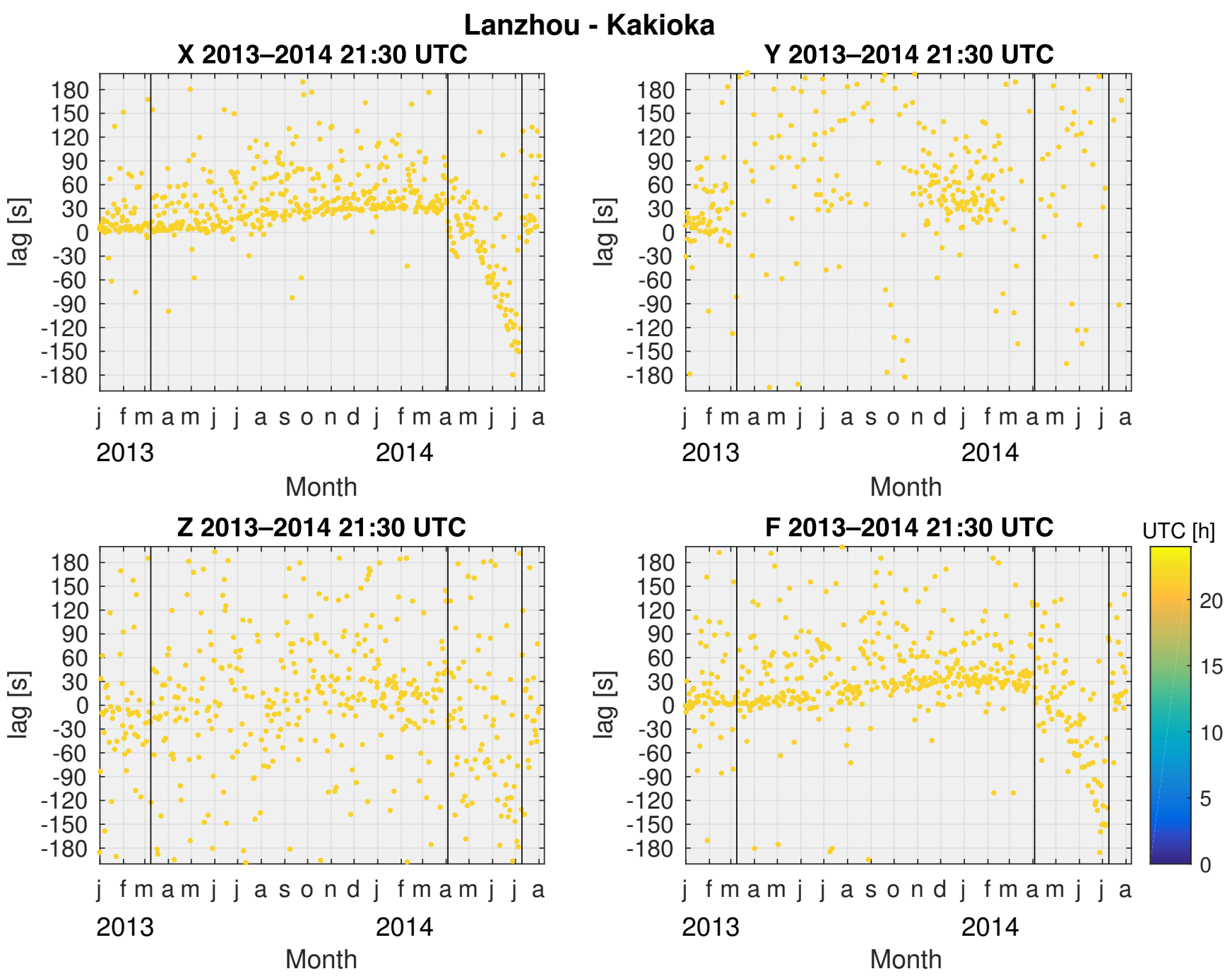

Figure 22. Time lags calculated every day during 1 hour around 21:30 UTC comparing Lanzhou and Kakioka data for each component of the magnetic field X, Y, Z and F. The vertical lines indicate from left to right: the time when the GPS synchronization became unavailable, the time when the data logger was rebooted and the time when the GPS synchronization was re-established. 

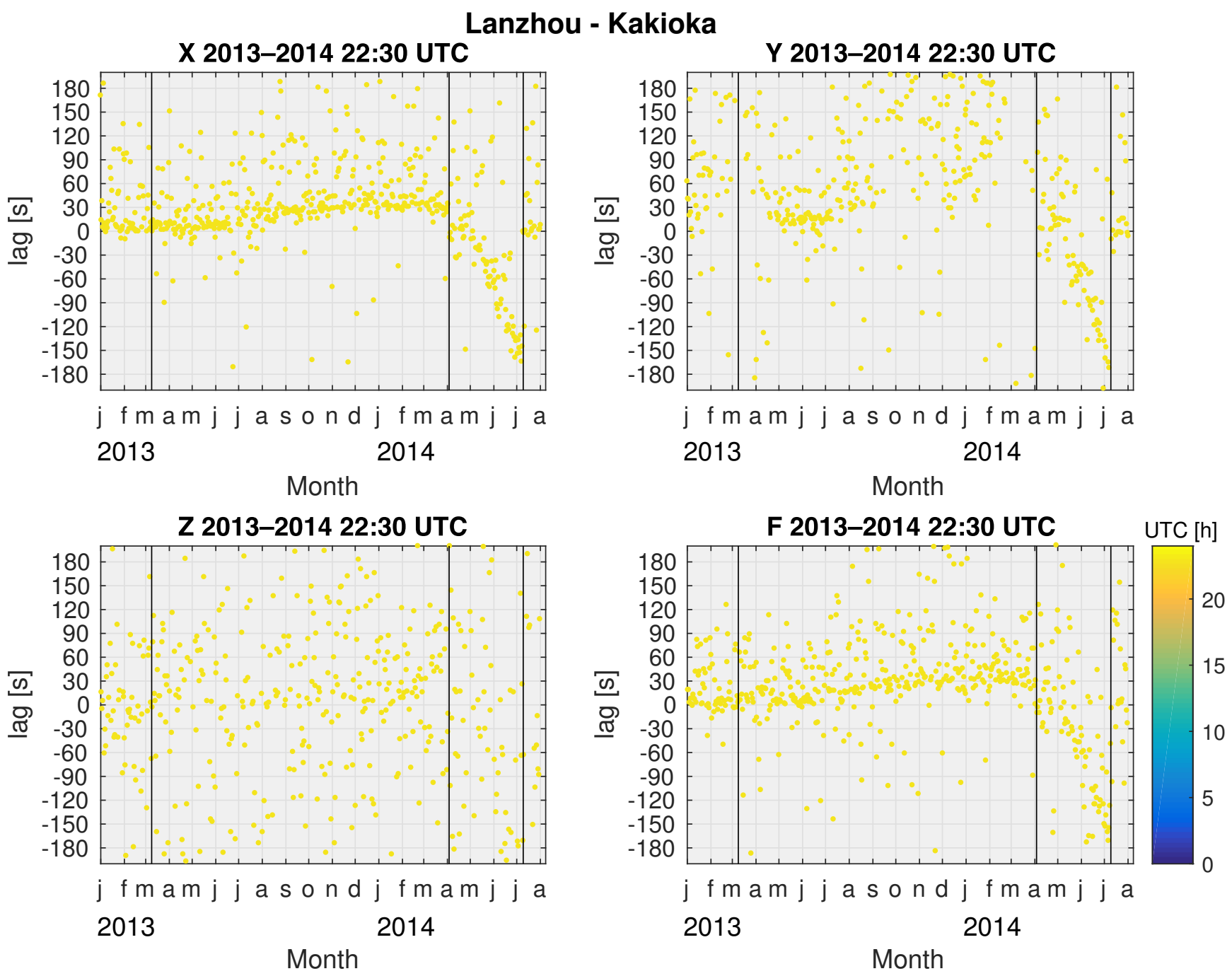

Figure 23. Time lags calculated every day during 1 hour around 22:30 UTC comparing Lanzhou and Kakioka data for each component of the magnetic field X, Y, Z and F. The vertical lines indicate from left to right: the time when the GPS synchronization became unavailable, the time when the data logger was rebooted and the time when the GPS synchronization was re-established. 

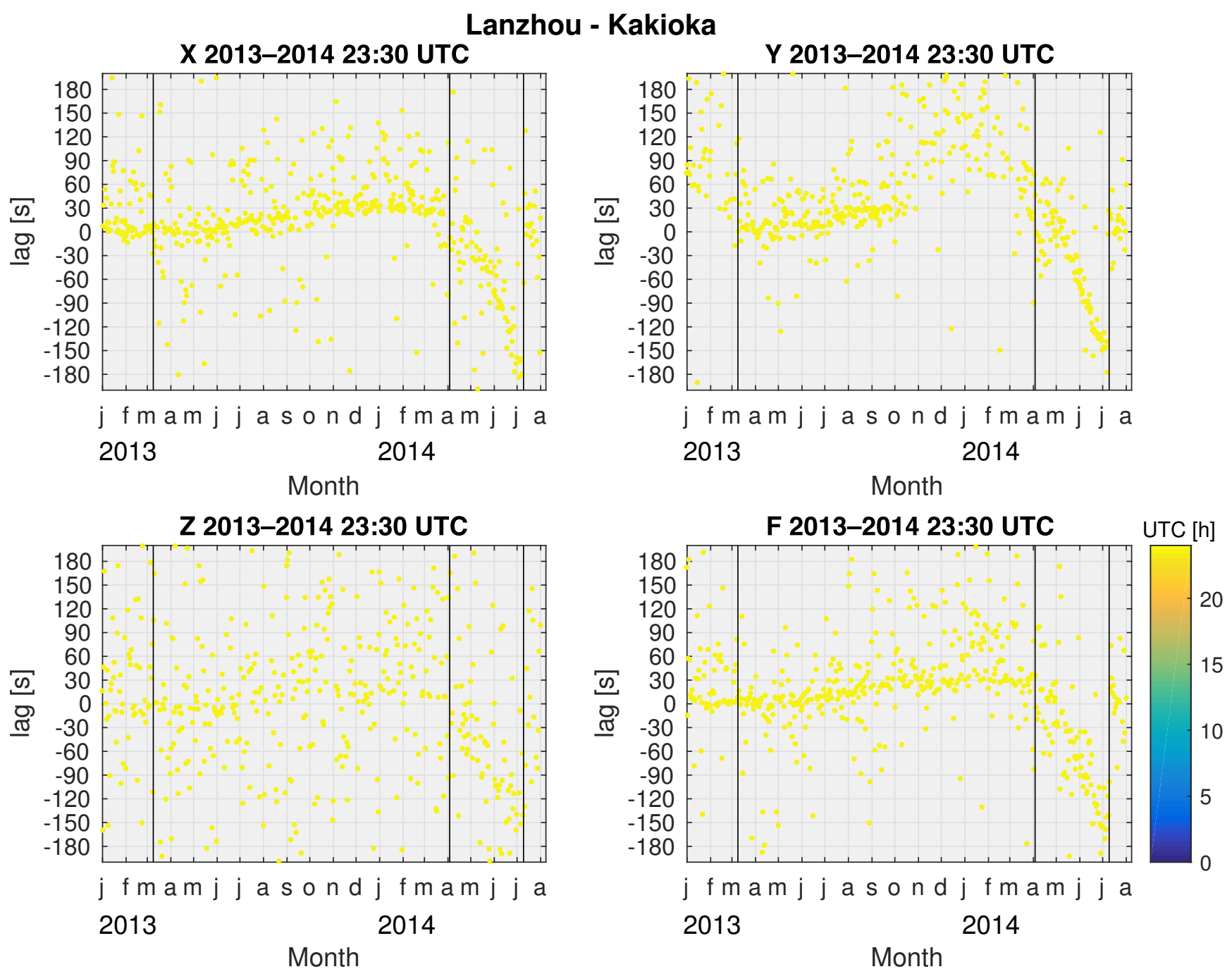

Figure 24. Time lags calculated every day during 1 hour around 23:30 UTC comparing Lanzhou and Kakioka data for each component of the magnetic field X, Y, Z and F. The vertical lines indicate from left to right: the time when the GPS synchronization became unavailable, the time when the data logger was rebooted and the time when the GPS synchronization was re-established. 

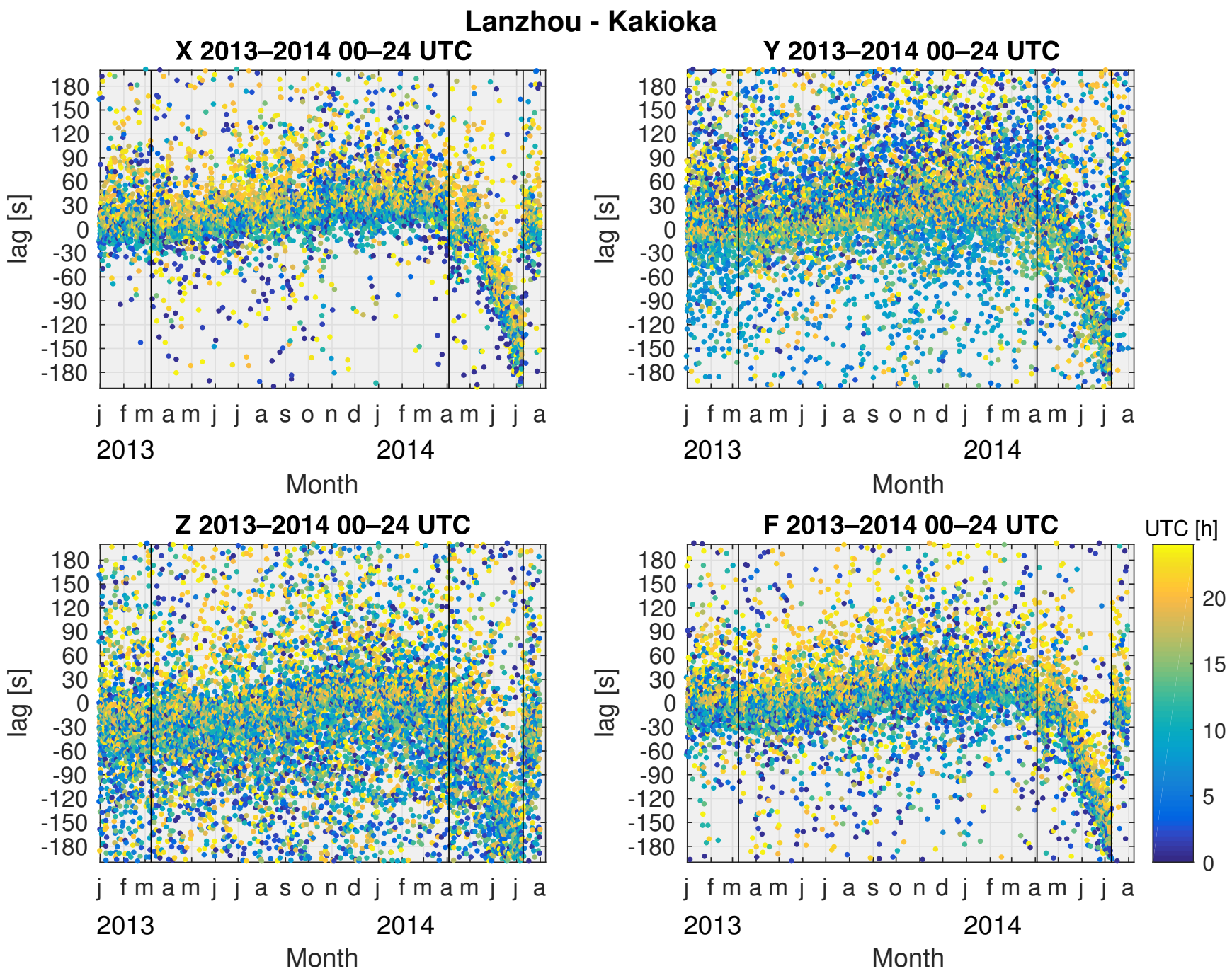

Figure 25. Time lags calculated every hour comparing Lanzhou and Kakioka data for each component of the magnetic field X, Y, Z and F. The vertical lines indicate from left to right: the time when the GPS synchronization became unavailable, the time when the data logger was rebooted and the time when the GPS synchronization was re-established. 